Théologiques

Théologiques

\title{
Croyances et contraintes sociales
}

\section{L'évolution du mouvement valentinien à la lumière du Traité tripartite (NH I,5) et du Dialogue du Sauveur (NH III,5)}

\section{Pierre Létourneau}

Volume 13, numéro 1, printemps 2005

Croire et croyances

URI : https://id.erudit.org/iderudit/012526ar

DOI : https://doi.org/10.7202/012526ar

Aller au sommaire du numéro

\section{Éditeur(s)}

Faculté de théologie et de sciences des religions, Université de Montréal

ISSN

1188-7109 (imprimé)

1492-1413 (numérique)

Découvrir la revue

Citer cet article

Létourneau, P. (2005). Croyances et contraintes sociales : l'évolution du mouvement valentinien à la lumière du Traité tripartite (NH I,5) et du Dialogue du Sauveur (NH III,5). Théologiques, 13(1), 79-94.

https://doi.org/10.7202/012526ar

\section{Résumé de l'article}

Le Dialogue du Sauveur (NH III,5), texte chrétien des premiers siècles demeuré totalement inconnu jusqu'à sa découverte dans le désert égyptien (Nag Hammadi) en 1945, est une pièce importante à verser au dossier de l'histoire du mouvement valentinien. Il atteste en effet d'un effort de rapprochement doctrinal avec la Grande Église, sans doute rendu nécessaire par l'uniformisation de la doctrine chrétienne autour du iv ${ }^{\mathrm{e}}$ siècle. On y trouve une révision accommodante des éléments centraux de la doctrine valentinienne, telles la création du cosmos par un démiurge inférieur et la tripartition de l'humanité. Évidemment, ce genre d'évolution doctrinale présuppose une notion souple de la « tradition de foi » et de la " quête de la vérité ».
Tous droits réservés (C Faculté de théologie et de sciences des religions, Université de Montréal, 2006
Ce document est protégé par la loi sur le droit d'auteur. L'utilisation des services d'Érudit (y compris la reproduction) est assujettie à sa politique d'utilisation que vous pouvez consulter en ligne.

https://apropos.erudit.org/fr/usagers/politique-dutilisation/ 


\section{Croyances et contraintes sociales \\ L'évolution du mouvement valentinien à la lumière du Traité tripartite (NH I,5) \\ et du Dialogue du Sauveur (NH III,5)}

Pierre LÉTOURnEAU

Faculté de théologie et de sciences des religions

Université de Montréal

Les Églises chrétiennes n'ont pas une tendance naturelle à modifier leur credo, le contenu fondamental et essentiel de la foi commune. Certes, la tradition évolue et s'enrichit au cours des siècles, notamment en ce qui a trait aux pratiques, aux lois morales, à la théologie. Mais le fondement de la foi, la "règle de la vérité », dirait Irénée de Lyon, demande stabilité et uniformité. Ne professe-t-on pas encore aujourd'hui la foi catholique d'après le Symbole des apôtres ou celui de Nicée-Constantinople? Or, certains textes valentiniens, découverts à Nag Hammadi il y a une soixantaine d'années, témoignent d'une modification progressive de la doctrine centrale du groupe, obéissant manifestement à une volonté de rapprochement avec la foi commune de la Grande Église ${ }^{1}$. Le présent article s'attardera d'abord à documenter cette évolution dans le Traité tripartite et le Dialogue du Sauveur, puis tentera d'éclairer les circonstances de cette transformation majeure de la doctrine valentinienne. Mais en tout premier lieu, il faut s'arrêter quelques instants à l'évolution de l'Église aux premiers siècles.

1. La «Grande Église » désigne le modèle romain, fondé sur le primat de Pierre et la succession apostolique, qui finira par s'imposer comme orthodoxie au IV siècle. 


\section{L'évolution de l'Église aux premiers siècles}

\subsection{La version classique: une tradition uniforme fondée sur la succession apostolique}

Le courant orthodoxe de l'Église, qui finit par s'imposer au cours du III et

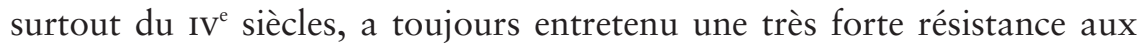
changements doctrinaux. Les quelques citations suivantes, reflétant l'enseignement officiel de l'Église, présentent la foi comme un dépôt sacré, transmis par les apôtres à l'Église, qui doit le préserver et le transmettre intact.

Tout d'abord, citons Irénée, évêque de Lyon, qui écrit vers 180 :

... ayant donc reçu cette prédication et cette foi, ainsi que nous venons de le dire, l'Église, bien que dispersée dans le monde entier, les garde avec soin, comme n'habitant qu'une seule maison, elle y croit d'une manière identique, comme n'ayant qu'une seule âme et qu'un même cœur, et elle les prêche, les enseigne et les transmet d'une voix unanime, comme ne possédant qu'une seule bouche. (Contre les hérésies, I,10,1 [Rousseau-Doutreleau 1979, 155-157])

Le modèle ecclésial qui se met en place durant la période patristique repose sur la transmission ininterrompue de la doctrine fondatrice: la révélation a été transmise aux apôtres qui l'ont transmise à leurs successeurs (les évêques), et ainsi de suite. Dans l'Église, la tradition de la vérité chevauche la succession apostolique.

Aussi la prédication apostolique, rapportée d'une façon spéciale dans les livres inspirés, devait-elle être conservée par une succession continuelle [d'évêques] jusqu'à la fin des temps. (Vatican II, Dei Verbum, 8 [Martin 1967, 106]) Ce qui est très important pour le Concile œcuménique, c'est que le dépôt sacré de la doctrine chrétienne soit conservé et présenté d'une manière plus efficace. [...] Il faut que cette doctrine certaine et immuable, qui doit être respectée fidèlement, soit approfondie et présentée de la façon qui répond aux exigences de notre époque. En effet, autre est le dépôt lui-même de la foi, c'est-à-dire les vérités contenues dans notre vénérable doctrine, et autre est la forme sous laquelle ces vérités sont énoncées, en leur conservant toutefois le même sens et la même portée. (Discours d'ouverture du concile Vatican II par Jean XXIII [1967, 585, 587]; voir aussi Gaudium et Spes 62,2)

"L'héritage sacré » de la foi (depositum fidei), contenu dans la Sainte Tradition et dans l'Écriture Sainte a été confié par les apôtres à l'ensemble de l'Église. [...] Pourtant, ce Magistère n'est pas au-dessus de la parole de Dieu, mais il la sert, n'enseignant que ce qui fut transmis, [...] et puise en cet unique 
dépôt de la foi tout ce qu'il propose à croire comme étant révélé par Dieu. (Catéchisme de l'Église catholique, chap. 2, art. 2, III, 84-86 [1993, 32-33])

On distingue mieux aujourd'hui tout ce que cette conception dogmatique doit aux stratégies et outils forgés par les Pères des $\mathrm{II}^{\mathrm{e}}$ et $\mathrm{III}^{\mathrm{e}}$ siècles dans leur combat acharné contre l'hérésie (voir Le Boulluec 1985). On connaît également le support que lui a procuré l'histoire traditionnelle de l'Église, en grande partie tributaire de l'Histoire ecclésiastique d'Eusèbe de Césarée, visant à façonner une Église unifiée autour de la foi et de la succession apostoliques ${ }^{2}$, un christianisme à la hauteur du statut impérial que venait de lui conférer l'empereur Constantin. Mais il s'agit en grande partie d'une image déformante des trois premiers siècles d'existence du christianisme.

\subsection{La version étouffée: une diversité de christianismes primitifs, pas tous "orthodoxes"}

Les débuts du christianisme ont été beaucoup moins unifiés qu'on a voulu plus tard le faire croire pour des raisons apologétiques et hérésiologiques. La diversité des formes et des doctrines est nettement apparente dès les premières décennies, comme en témoignent les écrits du Nouveau Testament (voir Dunn 1977). Il semble même que dans plusieurs régions de l'Empire, les premières formes attestées de christianismes soient celles qui reçurent plus tard les étiquettes d' " hétérodoxie » ou d' " hérésie ${ }^{3}$ ».

Pour les Pères, là où l'on trouve l'hérésie, l'orthodoxie doit avoir précédé. L'hérésie est toujours vue comme une déviation par rapport à la doctrine originale (orthodoxie) transmise par les apôtres. En réalité, la forme romaine du christianisme ne s'imposera que progressivement au cours des trois ou quatre premiers siècles, et ce qu'on nomme aujourd'hui l'" orthodoxie » ne verra le jour qu'après l'époque de Constantin et au prix de difficiles luttes doctrinales qui alimenteront les grands conciles œcuméniques des $I V^{e}$ et $V^{e}$ siècles. Avant cette époque, aucun modèle ecclésial ne s'impose

2. L'histoire n'est jamais «neutre ». L'historien qui décrit ou raconte l'histoire modifie inévitablement son objet d'étude, ne serait-ce que par les questions qu'il pose à ses sources, par la motivation qui l'anime ou par l'éclairage qu'il entend apporter à la situation présente. Eusèbe n'y échappe pas. Voir Veyne 1971 ; Marrou 1975 ; Bourdé et Martin 1983.

3. Selon la thèse de W. Bauer (1971) qui, malgré une argumentation inégale, a porté des coups décisifs à la conception traditionnelle de l'histoire de l'Église (voir Le Boulluec 1985, 14). 
et la compréhension du mystère chrétien est un véritable chantier où se côtoient diverses théologies et maints systèmes doctrinaux, qui constituent autant d'efforts légitimes de la foi qui cherche à se comprendre.

Le gnosticisme $e^{4}$ chrétien apparaît et se développe au sein de cette diversité. Certaines formes peu développées se contentent de proposer une expérience mystique de rencontre du divin à l'intérieur de soi, comme dans la tradition de Thomas (Évangile selon Thomas, Livre de Thomas). Mais dès le II $^{\mathrm{e}}$ siècle, la gnose s'érige en système et se présente avec un récit mythique des origines, des pratiques rituelles et même sacramentelles, ainsi qu'un enseignement moral prônant l'ascèse et le rejet du monde. Avant la fin du siècle, la gnose valentinienne est déjà si bien implantée qu'elle représente, aux yeux de l'évêque de Lyon, une menace sérieuse pour la Grande Église ${ }^{5}$.

Menaçante, tout d'abord, est l'attitude gnostique face à la révélation. La vérité n'est pas derrière soi, comme un dépôt légué par les apôtres, mais « en avant» (certains diraient «à l'intérieur de soi »), comme un objet qu'il ne faut jamais cesser de chercher: "Jésus a dit: Que celui qui cherche ne cesse de chercher jusqu'à ce qu'il trouve; et quand il aura trouvé, il sera bouleversé, et, étant bouleversé, il sera émerveillé, et il règnera sur le Tout. » (Évangile selon Thomas, log. 2 [1985, 25]) Certains plaçaient même cette quête de la vérité au centre de la démarche salvifique, comme si le salut éternel ne dépendait pas de la mort rédemptrice du Seigneur, mais d'une compréhension sans cesse approfondie de la révélation: «Et il [Jésus] a dit: Celui qui trouvera l'interprétation de ces paroles ne goûtera pas de la mort.» (Évangile selon Thomas, log. 1 [1985, 25]); "Le Seigneur dit: "[Si vous avez comp]ris tout ce que je [vous] ai dit, vous serez im[morte]ls...» (Dialogue du Sauveur, 143,2-5 [Létourneau 2003, 97]) Inévitablement, une telle attitude face à la révélation pouvait parfois conduire à de réels excès et à un foisonnement de doctrines qui n'avaient plus rien de commun avec la foi commune, comme le reprochait Tertullien aux valentiniens: "Ont-ils conçu quelque trouvaille, ils se hâtent de donner le nom de révélation à leur divagation, celui de charisme à leur invention mensongère, gage non pas d'unité mais de divergence. » (Contre les Valentiniens, IV,4 [1980, 89])

4. On discute encore de sa nature exacte et surtout de ses origines. Voir Williams 1996 ; King 2003.

5. Nul besoin d'insister sur le fait que les valentiniens se considéraient comme membres à part entière de l'Église. Pour les gnostiques, la gnose n'est pas une négation de la foi commune, mais un dépassement, une prolongation, la forme la plus pure du christianisme. Voir la thèse de Koschorke (1978) et la critique de Mahé (1993, 66-67). 
Mais ce qui a le plus irrité les Pères dans la gnose valentinienne, ce qui a soulevé leurs plus virulentes attaques, c'est le dualisme gnostique, tant dans sa forme théologique, qui place au-dessus du Créateur un Dieu suprême et bon n'ayant rien à faire du monde créé, que dans sa forme anthropologique, répartissant l'humanité en êtres hyliques, psychiques (les chrétiens ordinaires) et pneumatiques (les valentiniens), et réservant la plénitude du salut aux seuls pneumatiques, les autres étant voués à la destruction ou à un salut inférieur, à l'extérieur du Plérôme (voir Vallée 1981).

Ces prolégomènes m'ont semblé utiles à l'intelligence de ce qui suit. Il importait de souligner que les valentiniens se sont toujours considérés comme membres à part entière de l'Église primitive, au sein de laquelle ils ont pu élaborer leur système, développer leur théologie et entretenir une conception distincte de la révélation, du moins jusqu'à ce qu'une certaine forme d'Église s'impose, assimile les formes déviantes et se sépare des groupes récalcitrants. C'est sans doute à ce moment que certains valentiniens ont senti l'urgence d'ajuster les éléments irritants de leur doctrine.

\section{La doctrine valentinienne classique}

L'enseignement original de Valentin n'a survécu qu'à travers quelques fragments cités par Clément d'Alexandrie (Stromates), Irénée de Lyon (Contre les hérésies) et Hippolyte de Rome (Refutatio ${ }^{6}$ ), ce qui est bien maigre. Certains sont prêts à lui attribuer la composition de l'Évangile de vérité, connu d'Irénée (Contre les hérésies, III,11,9) et dont une version copte fut découverte à Nag Hammadi, mais cela reste une hypothèse difficile à prouver. Dans ces conditions, il est préférable de se tourner vers la version du mythe valentinien élaborée par Ptolémée, disciple de Valentin, qui fait l'objet d'un exposé détaillé dans ce qu'il est convenu d'appeler la "Grande Notice» d'Irénée de Lyon (Contre les hérésies, I,1-9), et dont je fais ici un résumé.

1) Le Plérôme est constitué de 30 éons, ou entités spirituelles (ogdoade, décade, dodécade), émanés par paires androgynes à partir de la dyade originelle, le Père de toutes choses, appelé «Abîme », et sa conjointe «Silence ». Un drame va troubler l'harmonie du Plérôme du fait que seul «Intellect », le premier éon émané du Père, est à même de le connaitre. En périphérie, Sophia, le plus jeune des éons, va succomber à l'envie de connaître le Père et s'élancer vers celui-ci, jusqu'à ce qu'elle soit remise à sa place et que sa

6. Les textes sont introduits et traduits dans Layton 1987, 217-249. 
«tendance » désordonnée (enthumêsis) soit expulsée hors du Plérôme. Une fois que tous les éons auront été consolidés, ils vont émettre le "Sauveur » et son escorte d'anges pour qu'ils aillent hors du Plérôme s'occuper de l'émanation informe de Sophia, appelée Achamoth. Cette dernière produit la substance hylique (matière), la substance psychique et la substance pneumatique qui entreront dans la fabrication du cosmos et de l'humanité (voir I,1-4).

2) Achamoth façonne un «Démiurge » avec la substance psychique, un dieu inférieur qui ignore tout du monde spirituel au-dessus de lui. Celui-ci se fabrique alors un monde matériel (cosmos), puis fait l'homme avec la substance hylique, dans laquelle il insuffle une âme psychique. C'est à son insu qu'Achamoth dépose la semence spirituelle dans l'âme humaine, afin que cette semence informe puisse croître et recevoir une formation de gnose en vue de son salut (voir I,5).

3) Les êtres humains se répartissent en trois natures: les pneumatiques (ou gnostiques), qui seront nécessairement sauvés, les psychiques (les croyants ordinaires de l’Église), qui peuvent espérer un salut éternel, mais inférieur à celui des pneumatiques, seulement s'ils se laissent raffermir par la foi et les bonnes œuvres, et enfin les hyliques (le reste de l'humanité), qui seront finalement détruits avec le cosmos (voir I,6).

4) La conception du salut est relativement simple: toute la substance pneumatique doit retourner dans le Plérôme, véritable chambre nuptiale où les êtres pneumatiques s'uniront avec les anges du Sauveur pour former des syzygies éternelles, à l'exemple du Sauveur et d'Achamoth. Quant aux psychiques qui auront pratiqué les bonnes œuvres, ils auront droit au repos en compagnie du Démiurge à l'extérieur du Plérôme, dans le lieu laissé vacant par Achamoth et le Sauveur. Car rien de psychique ne peut pénétrer dans le Plérôme (voir I,7).

Cette doctrine, à quelques variantes près, représente l'âge d'or du mouvement valentinien, au temps de Ptolémée, Héracléon et Théodote, à la fin $\mathrm{du} \mathrm{II}^{\mathrm{e}}$ siècle et dans les premières décennies du III $^{\mathrm{e}}$ siècle.

\section{Une tradition instable, sujette aux changements}

L'œil attentif découvre toutefois dans le système de Ptolémée des apories qui pourraient être l'indice de modifications rapides et superficielles apportées à la doctrine du fondateur. Je ne m'attarderai ici qu'à la position irrégulière des êtres psychiques. Ce sont en effet les seuls dont le salut ne repose pas sur une prédestination naturelle, mais sur un usage adéquat du libre arbitre. Par surcroît, leur repos éternel hors de la chambre nuptiale laisse 
subsister à l'égard du Plérôme un élément d'instabilité que tout le processus salvifique, commencé à l'intérieur du Plérôme, avait précisément pour but d'éliminer. La saga des psychiques a donc tout d'une pièce rapportée dans le système.

Aussi est-il tout naturel de penser, suivant en cela Gilles Quispel, que la doctrine originale de Valentin n'envisageait que la destruction de l'ordre cosmique et l'intégration de tous les êtres pneumatiques dans le Plérôme ${ }^{7}$ (voir 1980, 124, 129-130). Or, une séparation aussi radicale ne pouvait être moralement supportable que dans une Église majoritairement composée de gnostiques (pneumatiques) : dans l'idée du maître, tous les psychiques devaient être considérés comme des pneumatiques en devenir. Mais la réalité ne fut pas ainsi, en raison des fortes résistances suscitées par le message valentinien. Voilà, sans doute, ce qui explique que les représentants de la branche occidentale du mouvement (Ptolémée, Héracléon) aient fini par admettre une possibilité de salut inférieur pour ceux qui demeurent psychiques, faisant une concession aux croyants ordinaires de l'Église, au prix cependant d'une incohérence dans le système.

\section{Le Traité tripartite, témoin des grands changements}

Grâce à la découverte du Traité tripartite (Codex Jung), dont la rédaction finale date vraisemblablement du $\mathrm{III}^{\mathrm{e}}$ siècle (voir Attridge et Pagels 1985, vol. 1, 178; Thomassen et Painchaud 1989, 20), on a pu constater l'ampleur des modifications apportées à la doctrine centrale du valentinisme en l'espace d'un siècle. En réaction aux critiques des Pères, mais aussi à la suite de controverses internes, on aurait voulu faire évoluer la doctrine valentinienne dans un sens plus acceptable par la Grande Église ${ }^{8}$. Voici, à grands traits, les

7. Ce qui, mutatis mutandis, n'est pas si différent de la doctrine johannique, voulant que seuls les engendrés de l'Esprit aient accès au monde d'en haut (Jn 3,3-8), alors que tous les autres, demeurés "terrestres ", meurent ici-bas à cause de leur incrédulité (8,21-31).

8. L'hypothèse, d'abord émise par les auteurs de l'editio princeps (Kasser et al. 19731975, vol. 1, 313-314), puis reprise comme clef herméneutique du traité dans l'édition américaine (Attridge et Pagels 1985, vol. 1, 190), a connu un grand succès, au point que Quispel lui-même s’y est finalement rangé (1996, 349). Pour sa part, Jean-Daniel Dubois a préféré chercher du côté de la philosophie moyen-platonicienne l'explication de la conception monadique du Père dans le Traité tripartite, mais son refus de souscrire à l'hypothèse d'une «christianisation » progressive de la doctrine valentinienne aurait besoin d'une argumentation plus solide (Dubois 1995, 151-164). 
principaux aménagements doctrinaux attestés par le Traité tripartite (d'après Attridge et Pagels 1985, vol. 1, 190; voir aussi Quispel 1996, 348-352):

1) Alors que Valentin et, après lui, Ptolémée considéraient le Père suprême comme une dyade composée d' «Abîme» et "Silence », le Traité tripartite le présente comme une monade. Même la tétrade Abîme-Silence et Intellect-Vérité du système classique apparaît désormais comme une trinité composée du Père, du Fils et de l’Église/Esprit saint.

2) La figure centrale du mythe n'est plus Sophia-Achamoth, cette figure féminine déchue qui est responsable de la crise affectant le Plérôme, mais le Logos asexué, dont toutes les actions s'avèrent être en accord avec la volonté du Père. Celui-ci a la volonté d'être connu, mais il veut aussi que cela se fasse par un processus d'apprentissage graduel. En un sens, donc, même les conditions qui ont rendu la chute possible sont voulues par le Père (voir Thomassen et Painchaud 1989, 39-40).

3) Par voie de conséquence, le cosmos trouve lui aussi sa source ultime dans la volonté du Père. Certes, il s'agit encore de la fabrication d'un Démiurge inférieur, mais ce créateur n'est plus affublé d'attributs négatifs, mais plutôt dépeint comme l'instrument du Logos (voir Traité tripartite, 100,30-35).

4) Il faut enfin souligner la tendance à mieux apprécier l'élément psychique, et les "psychiques » eux-mêmes. L'ouverture envers la Grande Église atteint un sommet: même les croyants ordinaires de l'Église entreront finalement au Plérôme avec les pneumatiques (voir 123,116-122; 132,814.20-28). Ptolémée et Héracléon étaient conciliants, mais pas à ce point: ils n'envisageant qu'un repos éternel hors du Plérôme en compagnie du Démiurge (voir Irénée de Lyon, Contre les Hérésies, I,7,1; Clément d'Alexandrie, Extraits de Théodote, 34,2).

Comme on l'aura remarqué, la conception monadique du Père, la version trinitaire des entités suprêmes, l'intervention du Logos dans la création et le salut, et enfin l'accession des psychiques à la plénitude du salut sont toutes des corrections qui réduisent l'écart entre la doctrine valentinienne classique et la doctrine de la Grande Église.

\section{Le Dialogue du Sauveur: à la limite du valentinisme}

Le Dialogue du Sauveur est un document assez étonnant, d'abord parce qu'il réunit, dans sa facture même, deux sources littéraires d'horizons fort différents, l'une issue de la tradition thomasienne (Évangile selon Thomas, Livre de Thomas), l'autre provenant d'un milieu valentinien (voir Létourneau 2003, 18-41). Ensuite parce qu'il ne sort jamais d'une zone grise où 
christianisme orthodoxe et gnosticisme se côtoient à mots couverts. Donc, on ne trouvera pas cet anticosmisme radical typique de la gnose, mais pas non plus de louanges à l'adresse du Créateur pour son œuvre. Le cosmos est un monde de pauvreté sous la domination des archontes qui retiennent l'homme captif, mais il n'en est pas moins l'œuvre du Père et de son Logos'. Par ailleurs, pas de trace d'un monde divin divisé en tétrades ou décades d'éons spirituels, ni de la chute tragique de Sophia hors du milieu divin; pourtant, Dieu habite un Plérôme supracosmique, et une semence spirituelle est bel et bien tombée de là-haut pour se retrouver dans la profondeur du monde matériel. Enfin, les archontes et puissances diverses habitant le ciel cosmique et contrôlant le monde d'en bas ne sont pas le fruit de l'imaginaire gnostique, puisque Paul lui-même concède leur existence en affirmant qu'ils ont été vaincus par le Christ (voir Rm 8,38; 1Co 15,24; Ép 1,21; 6,12-13; Col 1,16;2,15). Certes, le Dialogue du Sauveur affirme l'origine pléromatique des élus, enseigne la présence intérieure du Dieu vivant et parle de la descente du Sauveur pour ramener les élus spirituels auprès du Père, mais en l'absence du mythe gnostique pour interpréter ces affirmations, on pourrait n'y voir qu'une adaptation des grands axes de la sotériologie johannique.

Pourtant, il faut bien reconnaître que la sotériologie du document, centrée sur ce qu'on pourrait appeler le «sacrement» de la chambre nuptiale, a des rapports très étroits avec la doctrine de l'école valentinienne ${ }^{10}$. À ma connaissance, seuls les valentiniens ont développé un tel schème de salut liant les thèmes du Plérôme, de l'origine céleste des élus, de la chute d'une semence déficiente et de l'existence de conjoints célestes qui reçoivent les élus et s'unissent à eux dans la chambre nuptiale (mystère de la syzygie). Les liens se resserrent davantage si nous ajoutons la mention de «la Mère du Tout », le titre « la Grandeur » appliqué au Père, ainsi que la désignation des élus et de leurs conjoints célestes par les termes «petits » et «grands » (voir la liste des termes techniques valentiniens dans Sagnard 1947, 627-660).

Devant ces formules et concepts, qui sont certes valentiniens, mais qui demeurent néanmoins des aspects secondaires du système, on a l'impression que le rédacteur du document a soigneusement évité de faire place aux éléments les plus radicaux de la doctrine valentinienne, ceux qui ont attiré

9. On ignore ce qui s'est passé pour que le cosmos passe sous la domination des archontes.

10. Ce qui suggère à Martin Krause que le Dialogue du Samveur provient d'une école valentinienne, malgré qu'il n'enseigne pas que les élus sont "sauvés par nature» (1977, 33-34). 
les foudres des hérésiologues. J'illustrerai cette hypothèse par deux exemples qui touchent à des aspects clefs de la doctrine valentinienne.

\subsection{Le thème de la création}

Le cosmos est un monde de pauvreté et déficient, actuellement sous le contrôle d'archontes terrifiants qui en gardent les issues et empêchent les âmes de s'échapper (Dialogue du Sauveur, 132,4-5 ; 139,17-18). Par ses nombreux attraits illusoires, le monde offre un repos trompeur et cause la perdition de plusieurs (141,16-19). Pourtant, c'est le Père qui a «établi le monde ${ }^{11}$ » et envoyé son Logos pour qu'il fasse jaillir la vie et tout ce que la Terre a besoin de génération en génération (129,20-130,23; 133,5-15). L'affirmation ne laisse de surprendre. On aurait plutôt imaginé que le monde soit l'œuvre d'un démiurge méchant ou, à tout le moins, ignorant, ce qui aurait été cohérent avec une telle conception du monde.

Ce n'est d'ailleurs pas le seul accroc à la logique du système cosmologique. Lorsque, un peu plus loin, il est répété que le Père a établi le monde, ce n'est plus le Logos qui l'assiste, mais «la Mère du Tout ${ }^{12}$ »(144,8-12). Puisque les deux énoncés font manifestement référence au même événement, il est légitime de songer à l'existence de deux versions du mythe cosmogonique derrière le Dialogue du Sauveur, ou mieux, d'envisager une correction apportée à un système qui ne considérait, à l'origine, que l'intervention créatrice de Sophia-Achamoth, la Mère déchue du Plérôme. Ce récit mythique a, du reste, laissé une autre trace dans notre document, là où il est fait mention des éons supérieurs et inférieurs qui ont dispersé la lumière dans le firmament et qui contrôlent, par conséquent, toutes les œuvres du monde matériel (131,5-16).

Il importe ici de souligner que le rédacteur ne s'est pas contenté de passer sous silence le mythe cosmogonique, en particulier la chute de Sophia et la création par le Démiurge, comme d'autres traités valentiniens, dits exo-

11. L'expression n'est pas totalement claire. On peut y lire que le Père a lui-même créé le monde, comme en Gn 1, ou simplement que la création du monde correspond à sa volonté, comme l'affirme aussi le Traité tripartite.

12. Il s'agit d'Achamoth, la Sophia inférieure, Mère du Tout, à un titre particulier dans le système valentinien, puisque c'est elle qui forme le Démiurge et agit à travers lui lorsqu'il crée le monde et façonne l'homme (voir Irénée de Lyon, Contre les hérésies, I,5,6 ; 8,4 ; Hippolyte, Refutatio, VI,33,1 ; Clément d'Alexandrie, Extraits de Théodote, 47,$2 ; 49 ; 53,4)$. 
tériques, l'ont fait (p. ex. la Lettre à Flora, l'Évangile de vérité et le Commentaire sur Jean d'Héracléon). Il n'a pas non plus suivi la piste du Traité tripartite en se limitant à rapatrier l'action d'un démiurge inférieur sous la volonté commune du Logos et du Père. En éliminant toute trace du Démiurge, le Dialogue du Sauveur affiche une version différente, «revue et corrigée » de la création, une version qui s'accorde avec l'élément premier du credo orthodoxe: le Père, Créateur du ciel et de la terre. Le remplacement de la Sophia coupable par le Logos obéissant va dans le même sens.

\subsection{La tripartition de l'humanité et le salut des psychiques}

L'anthropologie valentinienne classique, avec sa répartition de l'humanité entre hyliques, psychiques et pneumatiques, a traditionnellement servi de support au concept d'une rétribution différenciée pour chacune des trois classes d'humains, que cette tripartition soit conçue comme ontologique, et par conséquent déterministe, ou qu'elle soit l'actualisation de l'une ou l'autre potentialité commune, en réponse à la révélation ${ }^{13}$. Même le Traité tripartite, qui fait d'importantes concessions aux psychiques de la Grande Église, conserve le principe différenciateur de l'humanité, puisque la distinction entre les psychiques et les pneumatiques vaut pour tout le temps de l'oikonomia et ne sera abolie qu'à la fin des temps, lors de l'entrée finale et définitive dans le Plérôme.

Or, le Dialogue du Sauveur pousse plus loin encore la correction doctrinale. Ici, c'est toute l'âme qui doit s'élever dans la hauteur, soulevée en quelque sorte par l'esprit (pneuma) venu s'y loger $(128,15-23)$. En termes clairs, l'âme et l'esprit sont sauvés conjointement (147,20-22), ce qui rend caduque et inutile la distinction provocatrice entre l'élection et la vocation, c'est-à-dire entre les gnostiques et les croyants ordinaires, d'où l'absence dans le traité de la célèbre tripartition de l'humanité.

Il est intéressant de constater l'amélioration progressive du sort final de l'élément psychique en passant de Valentin à Ptolémée, puis au Traité tripartite et au Dialogue du Sauveur. Chez Valentin, les psychiques n'existent pas au plan sotériologique: ou bien ils deviennent des pneumatiques, ou bien ils sont détruits avec les êtres hyliques. Chez Ptolémée et dans le Traité tripartite, les psychiques ont désormais droit au salut, d'abord un salut inférieur

13. Voir Létourneau 1993, 418-424 ; Schottroff 1969; Pagels 1973; Aland 1977, 113 ; Pagels 1974, 35-53. 
hors du Plérôme, puis un salut plénier au même titre que les pneumatiques. Dans le Dialogue du Sauveur, c'est la distinction elle-même qui disparait, puisque l'Église comprend indistinctement les psychiques et les pneumatiques, à l'image de l'unité âme-pneuma à l'intérieur de l'homme. La différence entre croyants au sein de l'Église est abolie.

\section{Quelques conclusions embryonnaires}

1) Constater ce déplacement doctrinal vers la théologie de la Grande Église est une chose, l'expliquer en est une autre. Recourir à l'hypothèse d'un écrit de propagande missionnaire, un document épuré, comme dans le cas de la Lettre à Flora de Ptolémée, n'est pas approprié, puisque le Dialogue du Sauveur ne présente aucun argument visant à convertir ou à convaincre des non initiés. Du reste, si tel avait été le but de l'ouvrage, l'auteur aurait pu se contenter d'omettre simplement le mythe cosmogonique, comme Ptolémée l'a fait. Or, dans notre texte, il ne s'agit pas d'un simple silence, mais d'un véritable ajustement de la doctrine.

Je crois que l'attitude envers l'élément psychique constitue un indice révélateur au plan sociologique. De fait, la place de l'élément psychique dans le système valentinien reflète essentiellement le positionnement du groupe à l'intérieur de l'Église. On pourrait croire que dans le système de Valentin, il n’y a pas de tension : l'Église véritable (eschatologique ?) est composée uniquement des pneumatiques et tous les croyants doivent cheminer jusqu'à ce stade. Le système de la seconde génération, à l'inverse, manifeste un état de tension élevée, alors que deux catégories de croyants subsistent éternellement : les valentiniens se perçoivent comme une élite supérieure, tandis que la masse des croyants ordinaires vont demeurer inférieurs et n'auront jamais accès à la plénitude du salut. Nous sommes sans doute au stade du rejet de la prédication valentinienne, mais à l'époque où la tradition pétrinienneromaine ne s'est pas encore imposée à toute l'Église.

Suivant ce cadre explicatif, le Traité tripartite témoignerait au plan doctrinal des premiers efforts de réconciliation, alors que le groupe minoritaire a de la difficulté ou ne peut plus assurer sa place à l'intérieur de l'Église, au moment où celle-ci s'unifie autour de la tradition romaine du christianisme. Dans le Dialogue du Sauveur, la tension a disparu et tous les croyants partagent le même statut. La doctrine du groupe n'a plus rien d'irritant pour le reste de l'Église; un rédacteur final a même fait place à l'aspect sacrificiel du salut opéré par le Fils (121,18-22). Ce qui reste de la sotériologie valentinienne traditionnelle, à savoir l'union mystique des croyants avec les anges 
(pneumatiques) du Sauveur dans la chambre nuptiale, pouvait encore avoir droit de cité à côté des nombreuses autres images du salut à cette époque.

Nul besoin d'insister sur le fait que ce genre de modifications sur des points majeurs du credo valentinien témoigne des fortes contraintes sociales qui ont dû affecter la vie du groupe, au point où l'accommodement doctrinal paraissait un moindre mal.

2) Encore faut-il une conception de la foi et de la révélation qui permette ce genre d'altération majeure. Un tel recul doctrinal aurait été plus difficile, voire impossible, dans le système romain fondé sur la succession apostolique et la transmission fidèle d'une règle de foi héritée des premiers croyants. Mais quand la vérité fait l'objet d'une constante recherche, quand le salut est un droit acquis par la simple présence intérieure de l'Esprit, la révélation peut plus facilement s'accommoder du devenir historique.

De plus, le morcellement géographique et la forte composante individuelle (recherche intérieure) du gnosticisme pouvaient entraîner une certaine fragilité sociale, et peut-être aussi une plus grande malléabilité doctrinale.

3) La nature même de la doctrine a sans doute aussi quelque chose à voir avec cette facilité d'adaptation. La forme mythique, qui prend l'allure d'un long récit, laisse beaucoup plus d'emprise aux transformations que les formules dogmatiques brèves et stables. Du reste, la gnose s'apparente plus à une quête d'intelligence qu'à la confession dogmatique de foi.

\section{Références}

Aland, B. (1977), «Erwählungstheologie und Menschenklassenlehre », dans M. Krause, dir., Gnosis and Gnosticism, Leiden, Brill (Nag Hammadi Studies 8), p. 148-181.

Attridge, H.W. et E.H. Pagels (1985), «The Tripartite Tractate (I,5)», dans H.W. Attridge, dir., Nag Hammadi Codex I, Leiden, Brill (Nag Hammadi Studies 22-23), p. 159-337 (I), 217-497 (II).

Bauer, W. (1971) [allemand 1964², 1934], Orthodoxy and Heresy in Earliest Christianity / trad. par Philadelphia Seminar on Christian Origins, édité et complété par R.A. Kraft et G. Krodel, Philadelphia, Fortress Press.

Bourdé, G. et H. Martin (1983), Les écoles historiques, Paris, Seuil (Points. Histoire).

- Catéchisme de l'Église catholique (1993) [latin 1992], Ottawa, Service des Éditions de la Conférence des Évêques catholiques du Canada. 
Dubois, J.-D. (1995), «Le Traité Tripartite (NHC I,5) et l'histoire de l'école valentinienne ", dans A. LE BoullueC, dir., La controverse religieuse et ses formes, Paris, Cerf (Patrimoines. Religions du Livre), p. 151-164.

DunN, J.D.G. (1977), Unity and Diversity in the New Testament: An Inquiry Into the Character of Earliest Christianity, Philadelphia, Westminster Press.

Évangile selon Thomas (1985) / présentation, traduction et commentaires de É. Gillabert, P. Bourgeois et Y. Haas, Paris, Dervy-Livres.

Jean XXVIII (1967), "Discours pour l'ouverture solennelle du Concile (11 octobre 1962)", dans P.-A. Martin, dir., Vatican II. Les seize documents conciliaires. Texte intégral, Montréal / Paris, Fides (La pensée chrétienne), p. 581-591 [traduction tirée de La Documentation catholique, 4 (1962), col. 1377-1386].

Kasser, R. et al. (1973-1975), Tractatus Tripartitus, Berne, Francke.

KInG, K.L. (2003), What is Gnosticism? Cambridge, Belknap Press.

Koschorke, K. (1978), Die Polemik der Gnostiker gegen das kirchliche Christentum, Leiden, Brill (Nag Hammadi Studies 12).

Krause, M. (1977), "Der Dialog des Soter in Codex III von Nag Hammadi », dans Gnosis and Gnosticism, Leiden, Brill (Nag Hammadi Studies 8), p. 13-34.

Layton, B. (1987), The Gnostic Scriptures, Garden City, Doubleday.

Le Boulluec, A. (1985), La notion d'hérésie dans la littérature grecque, II III siècles, t. I: De Justin à Irénée, Paris, Études augustiniennes.

LÉTourneau, P. (1993), "Éthique et nature humaine dans la gnose valentinienne ", Église et théologie, 24, p. 413-441.

- (2003), Le Dialogue du Sauveur (NH III,5), Québec / Louvain / Paris, Presses de l'Université Laval / Peeters (Bibliothèque copte de Nag Hammadi, section «Textes » 29).

MaнÉ, J.-P. (1993), «L'élitisme gnostique et la souillure de la Grande Église d'après les écrits de Nag Hammadi ", dans H.D. Altendorf et al., Orthodoxie et hérésie dans l'Église ancienne, Genève (Cahiers de la Revue de théologie et de philosophie 17), p. 65-95.

Marrou, H.-I. (1975), De la connaissance historique, Paris, Seuil (Points. Histoire).

Martin, P.-A., dir. (1967) [latin 1966], "Constitution dogmatique de Divina Revelatione (Dei Verbum) » / trad. par G. Blond, et Y. Congar, 
dans Vatican II. Les seize documents conciliaires. Texte intégral, Montréal / Paris, Fides (La pensée chrétienne), p. 97-120.

Pagels, E.H. (1973), The Johannine Gospel in Gnostic Exegesis: Heracleon's Commentary on John, Nashville / New York, Abingdon Press.

- (1974), «Conflicting Versions of Valentinian Eschatology : Irenæus’ Treatise vs. the Excerpts from Theodotus ", Harvard Theological Review, 67, p. 35-53.

Quispel, G. (1980), «Valentinian Gnosis and the Apocryphon of John", dans B. Layton, dir., Rediscovery of Gnosticism, t. I: The School of Valentinus, Leiden, Brill (Studies in the History of Religions. Supplements to Numen 41).

- (1996), "The Original Doctrine of Valentinus the Gnostic », Vigiliae Christianae, 50, p. 327-352.

Rousseau, A. et L. Doutreleau (r979), Irénée de Lyon. Contre les hérésies, Livre I, Paris, Cerf (Sources chrétiennes 264).

SAGNARD, F.-M.-M. (1947), La gnose valentinienne et le témoignage de Saint Irénée, Paris, Vrin (Études de philosophie médiévale 36).

Schotтroff, L. (1969), «Animæ naturaliter salvandæ. Zum Problem der himmlischen Herkunft des Gnostikers ", dans W. Eltester, dir., Christentum und Gnosis, Berlin, Töpelmann, p. 65-97.

Tertullien (1980), Contre les Valentiniens / édition de J.-C. Fredouille, Paris, Cerf (Sources chrétiennes 280).

Thomassen, E. et L. Painchaud (1989), Le Traité tripartite (NH I,5), Québec, Presses de l'Université Laval (Bibliothèque copte de Nag Hammadi, section «Textes» 19).

Vallée, G. (1981), A Study in Anti-Gnostic Polemics: Irenceus, Hippolytus, and Epiphanius, Waterloo, Wilfrid Laurier University Press (Studies in Christianity and Judaism 1).

Veyne, P. (1971), Comment on écrit l'histoire. Texte intégral, Paris, Seuil (Points. Histoire).

Williams, M. (1996), Rethinking Gnosticism: An Argument for Dismantling a Dubious Category, Princeton, Princeton University Press.

\section{Résumé}

Le Dialogue du Sanveur (NH III,5), texte chrétien des premiers siècles demeuré totalement inconnu jusqu'à sa découverte dans le désert égyptien (Nag Hammadi) en 1945, est une pièce importante à verser au dossier de l'histoire du mouvement 
valentinien. Il atteste en effet d'un effort de rapprochement doctrinal avec la Grande Église, sans doute rendu nécessaire par l'uniformisation de la doctrine chrétienne autour du IV $v^{e}$ siècle. On y trouve une révision accommodante des éléments centraux de la doctrine valentinienne, telles la création du cosmos par un démiurge inférieur et la tripartition de l'humanité. Évidemment, ce genre d'évolution doctrinale présuppose une notion souple de la «tradition de foi » et de la «quête de la vérité ».

\section{Abstract}

The Dialogue of the Savior (NH III,5), an ancient Christian text completely unknown until its discovery in the Egyptian desert (Nag Hammadi) in 1945, provides evidence of a will of moving closer to the Great Church in the Valentinian movement. As the Church was engaged in a process of unification in the fourth century, some among the Valentinians would have revised in an accommodating way important doctrinal features, such as the creation of the cosmos by an inferior god and the division of humanity in hylics, psychics and pneumatics. This kind of doctrinal revision is made possible by a different notion of truth and tradition. 